Article

\title{
Gravitational Contribution to the Heat Flux in a Simple Dilute Fluid: An Approach Based on General Relativistic Kinetic Theory to First Order in the Gradients
}

\author{
Dominique Brun-Battistini ${ }^{1, *, \dagger}$, Alfredo Sandoval-Villalbazo ${ }^{1, \dagger}$ and \\ Ana Laura Garcia-Perciante ${ }^{2}$ \\ 1 Departamento de Fisica y Matematicas, Universidad Iberoamericana Ciudad de Mexico, \\ Prolongacion Paseo de la Reforma 880, Mexico D.F. 01219, Mexico; alfredo.sandoval@ibero.mx \\ 2 Departamento de Matematicas Aplicadas y Sistemas, Universidad Autonoma Metropolitana-Cuajimalpa, \\ Prolongacion Vasco de Quiroga 4871, Mexico D.F. 05348, Mexico; algarcia@correo.cua.uam.mx \\ * Correspondence: dominique.brun@ibero.mx; Tel.: +52-55-5950-4071 \\ + These authors contributed equally to this work.
}

Received: 11 August 2017; Accepted: 9 October 2017; Published: 28 October 2017

\begin{abstract}
Richard C. Tolman analyzed the relation between a temperature gradient and a gravitational field in an equilibrium situation. In 2012, Tolman's law was generalized to a non-equilibrium situation for a simple dilute relativistic fluid. The result in that scenario, obtained by introducing the gravitational force through the molecular acceleration, couples the heat flux with the metric coefficients and the gradients of the state variables. In the present paper it is shown, by explicitly describing the single particle orbits as geodesics in Boltzmann's equation, that a gravitational field drives a heat flux in this type of system. The calculation is devoted solely to the gravitational field contribution to this heat flux in which a Newtonian limit to the Schwarzschild metric is assumed. The corresponding transport coefficient, which is obtained within a relaxation approximation, corresponds to the dilute fluid in a weak gravitational field. The effect is negligible in the non-relativistic regime, as evidenced by the direct evaluation of the corresponding limit.
\end{abstract}

Keywords: relativity; kinetic theory; fluid mechanics; heat conduction

\section{Introduction}

The problem of calculating the heat flux in a simple dilute relativistic fluid due to a gravitational field can be approached from different perspectives. In 1930, Richard C. Tolman considered such system in an equilibrium situation and showed that a gravitational field can balance a temperature gradient, leading to a vanishing heat flux; this is known as Tolman's law [1]. Several decades later, in 2012, an expression for the heat flux in the presence of a linearized gravitational field was established in a non-equilibrium situation and Tolman's law was recovered when the equilibrium limit is attained [2].

On the other hand, in reference [3] the heat flux was calculated using a Schwarzschild metric with isotropic coordinates within the framework of general relativistic kinetic theory, concluding that the contribution of the gravitational field vanishes. In that work, it was suggested that the effect obtained in reference [2] may be traced back to a metric factor that has not been considered in the equilibrium distribution function.

In the present paper it is shown that the gravitational contribution to the heat flux prevails when assuming structureless particles moving along geodesics. The calculation is performed in a local Minkowsky space-time and the thermodynamic forces appear in a hydrodynamic scale [4]. These important conceptual features improve the formalism presented in reference [2]. Moreover, thermodynamic forces corresponding 
to the gravitational field are established through the covariant derivatives of the hydrodynamic velocity present in the formalism.

Another approach to this problem was presented in 1984 by Wodarzik [5]. In that work the heat flux is expressed using the corresponding Eckart constitutive equation [6]. It is relevant to mention that the well known hydrodynamic generic instabilities arise if this type of coupling is assumed [7]. Moreover, in that work it is assumed that the fluid as a bulk moves following geodesics, an argument that can be questioned as individual molecules follow geodesics [4], while stresses deviate the bulk from this type of dynamics. On the other hand, in the present paper, we introduce the formal kinetic theory definition of heat flux in the Navier-Stokes regime with no hydrodynamic acceleration present in the constitutive equation. According to the assumptions of linear irreversible thermodynamics, heat flux is driven by all vector gradients present in the system such that it is coupled with $\nabla T, \nabla n$ (see references [2,7-9]) and, in the presence of a gravitational field, to the gravitational potential gradient.

In order to establish the heat flux following the approach described above, the rest of this paper is divided as follows. In Section 2, we review a few concepts regarding the Boltzmann equation in the general relativistic regime. In Section 3, the first order in the gradients approximation to the distribution function $\left(f^{(1)}\right)$ is obtained, following the general relativistic kinetic theory formalism in the BGK approximation within the Chapman-Enskog expansion. The calculation of the heat flux is shown in Sections 4 and 5 is devoted to final remarks.

\section{Basic Formalism: Relativistic Fluids}

\subsection{Basic Elements of General Relativity and the Schwarzschild Metric}

A simple, non-degenerate gas is considered in a curved space-time where the line element (arc length) is expressed as $[4,10,11]$ :

$$
d s^{2}=g_{\mu v} d x^{\mu} d x^{v},
$$

where $g_{\mu \nu}$ is the metric tensor. Different metrics may be obtained from Einstein's field equations, depending on the symmetries present in the system to be analyzed. For a Schwarzschild metric, which has the property of being spherically symmetric and static, the line element is given by (with a signature +++-$)$ :

$$
d s^{2}=\frac{1}{\left(1-\frac{2 G M}{c^{2} \tilde{r}}\right)} d \tilde{r}^{2}+\tilde{r}^{2}\left(d \theta^{2}+\sin ^{2} \theta d \varphi^{2}\right)-\left(1-\frac{2 G M}{c^{2} \tilde{r}}\right)\left(d x^{4}\right)^{2},
$$

with $G$ being the gravitational constant, $c$ the speed of light, $M$ the total mass, source of the gravitational field, and $r, \theta$, and $\varphi$ the spherical coordinates. As in reference [3], an isotropic Schwarzschild metric is used [12], for which the substitution $\tilde{r}=r\left(1+\frac{G M}{c^{2} r}\right)$ is introduced in Equation (2). Considering $\Phi(r)=\frac{G M}{r}$, the gravitational potential, the Newtonian limit of the Schwarzschild metric, which corresponds to the weak field approximation $\left(\frac{\Phi}{c^{2}} \ll 1\right)$ reads:

$$
d s^{2}=\left(1+\frac{2 \Phi}{c^{2}}\right)\left(d x^{2}+d y^{2}+d z^{2}\right)-\left(1-\frac{2 \Phi}{c^{2}}\right)\left(d x^{4}\right)^{2}
$$

In order to perform the relevant calculations in the following sections it is convenient to recall that in general relativity, the four-velocity is the derivative of the position four-vector with respect to the arc length, that is:

$$
v^{\mu}=c \frac{d x^{\mu}}{d s}
$$


Also, in the following sections use of the Christoffel symbols is made, with the usual definition being:

$$
\Gamma_{\alpha \beta}^{\mu}=\frac{\tilde{g}^{\mu v}}{2}\left(\frac{\partial \tilde{g}_{\alpha v}}{\partial x^{\beta}}+\frac{\partial \tilde{g}_{\beta v}}{\partial x^{\alpha}}-\frac{\partial \tilde{g}_{\alpha \beta}}{\partial x^{v}}\right)
$$

It must be noticed that the only non-vanishing symbols for this metric are the following:

$$
\begin{gathered}
\Gamma_{11}^{1}=\Gamma_{21}^{2}=\Gamma_{31}^{3}=\Gamma_{12}^{2}=\Gamma_{13}^{3}=\frac{\Phi^{\prime}}{c^{2}\left(1+\frac{2 \Phi}{c^{2}}\right)}, \\
\Gamma_{22}^{1}=\Gamma_{33}^{1}=\Gamma_{44}^{1}=-\frac{\Phi^{\prime}}{c^{2}\left(1+\frac{2 \Phi}{c^{2}}\right)}, \\
\Gamma_{14}^{4}=\Gamma_{41}^{4}=\frac{\Phi^{\prime}}{c^{2}\left(-1+\frac{2 \Phi}{c^{2}}\right)} .
\end{gathered}
$$

\subsection{Boltzmann's General Relativistic Equation}

Relativistic kinetic theory has been successfully applied to the study of high temperature astrophysical and cosmological fluids $[13,14]$. When the thermal energy of a single mass particle $(k T)$ is comparable to its rest mass $\left(m c^{2}\right)$, the ordinary Maxwellian distribution function must be modified in order to be consistent with the tenets of the relativity theory. Decades after the pioneering works of Jüttner [15], a thorough revision of its validity was performed by Cubero, Dünkel and other authors [16]. Boltzmann's equation describes the evolution of the single-particle distribution function and is expressed as follows $[9,10]$ :

$$
\dot{f}=J\left(f f^{\prime}\right),
$$

where $J\left(f f^{\prime}\right)$ is the collisional kernel. Since $f$ is a function of the position $\left(x^{\mu}\right)$ and velocity $\left(v^{\mu}\right)$ four-vectors, $\dot{f}$ can be written as:

$$
\dot{f}=\frac{\partial f}{\partial x^{\mu}} \dot{x}^{\mu}+\frac{\partial f}{\partial v^{\mu}} \dot{v}^{\mu}
$$

In the special relativistic regime, a dot denotes the total proper time $(\tau)$ derivative in a flat space-time. On the other hand, in a general relativistic scenario, $\dot{f}$ corresponds to the arc length derivative $\left(\dot{f}=\frac{d f}{d s}\right)$, with $s$ being the arc length [4]. Also in this approach, we have $\dot{v}^{\mu}=v^{\alpha} v_{; \alpha}^{\mu}=$ $v^{\alpha}\left(\frac{\partial v^{\mu}}{\partial x^{\alpha}}+\Gamma_{\alpha \beta}^{\mu} v^{\beta}\right)$.

In this paper, particles are assumed to lack structure (they do not have internal degrees of freedom) so that as a consequence of the field equations, the acceleration $\dot{v}^{\mu}=\frac{d v^{\mu}}{d s}$ is zero, and the molecules move following geodesics, i.e.,:

$$
\frac{d^{2} x^{\mu}}{d s^{2}}+\Gamma_{\alpha \beta}^{\mu} \frac{d x^{\alpha}}{d s} \frac{d x^{\beta}}{d s}=0 .
$$

The Boltzmann's equation can thus be written as:

$$
v^{\mu} f_{, \mu}=\frac{\partial f}{\partial x^{\mu}} v^{\mu}=J\left(f f^{\prime}\right)
$$

where Equation (4) has been used. The collisional kernel can be modeled in a simple form using the BGK approximation [17], so that Equation (12) becomes:

$$
v^{\mu} f_{, \mu}=-\frac{f-f^{(0)}}{\tau_{c}}
$$

where $\tau_{c}$ is a relaxation time and $f^{(0)}$ the local equilibrium distribution function. 
In order to calculate the heat flux, the first order in the gradients correction to the distribution function $f^{(1)}$ must be obtained. Following the kinetic theory approach, Chapman-Enskog's method [10] is now used, such that a solution given by $f=f^{(0)}+f^{(1)}$ is assumed. By substituting this solution in Equation (13) and after simple algebraic manipulations, one obtains:

$$
f^{(1)}=-\tau_{c} \frac{\partial f^{(0)}}{\partial x^{\mu}} v^{\mu}
$$

The next step consists of introducing the functional hypothesis by means of which the factor $\frac{\partial f^{(0)}}{\partial x^{\mu}}$ is written in terms of the state variables $n$ (the local particle number density), $T$ (the local temperature) and $\mathcal{U}^{\mu}$ (the hydrodynamic fluid velocity) [8]:

$$
\frac{\partial f^{(0)}}{\partial x^{\mu}}=\frac{\partial f^{(0)}}{\partial n} \frac{\partial n}{\partial x^{\mu}}+\frac{\partial f^{(0)}}{\partial T} \frac{\partial T}{\partial x^{\mu}}+\frac{\partial f^{(0)}}{\partial \mathcal{U}^{\alpha}} \mathcal{U}_{; \mu}^{\alpha}
$$

The covariant derivative $\mathcal{U}_{; \mu}^{\alpha}$ introduced in Equation (15) is imperative to preserve its invariance. Indeed, the quotient rule leads to the use of the covariant derivative of the velocity field. It is important to notice that in the definition of the covariant derivative of this local variable:

$$
\mathcal{U}_{; \mu}^{\alpha}=\frac{\partial \mathcal{U}^{\alpha}}{\partial x^{\mu}}+\Gamma_{\mu \nu}^{\alpha} \mathcal{U}^{v}
$$

the second term will become a thermodynamic force.

The Jüttner (Maxwell-Boltzmann relativistic) function must be considered in order to establish the derivatives in Equation (15):

$$
f^{(0)}=\frac{n}{4 \pi c^{3} z K_{2}\left(\frac{1}{z}\right)} e^{\mathcal{U}^{\alpha} v_{\alpha} / z c^{2}}=\frac{n}{4 \pi c^{3} z K_{2}\left(\frac{1}{z}\right)} e^{-\frac{\gamma}{z}},
$$

where $K_{\ell}\left(\frac{1}{z}\right)$ is the modified Bessel function of the second kind of order $\ell$ and $z=k_{B} T / m c^{2}$ is the relativistic thermal parameter, with $k_{B}$ the Boltzmann constant. The invariant $\mathcal{U}^{v} v_{v}$ in the equilibrium distribution function in Equation (17) can be written in terms of the molecular speed when calculated in a reference frame where the spatial components of $\mathcal{U}^{v}$ vanish (comoving frame). In such a frame $\mathcal{U}^{4} \approx c$ in the scale in which our dilute fluid is describable by a Jüttner function (Equation(17)).

Substituting in Equation (15) the covariant derivative's definition (Equation (16)) and the corresponding partial derivatives of $f^{(0)}$ one obtains:

$$
\frac{\partial f^{(0)}}{\partial x^{\mu}}=f^{(0)}\left[\frac{1}{n} \frac{\partial n}{\partial x^{\mu}}+\left(1+\frac{\mathcal{U}^{v} v_{v}}{z c^{2}}-\frac{\mathcal{G}\left(\frac{1}{z}\right)}{z}\right) \frac{\partial T}{\partial x^{\mu}}+\frac{v_{\alpha}}{z c^{2}}\left(\frac{\partial \mathcal{U}^{\alpha}}{\partial x^{\mu}}+\Gamma_{\mu \nu}^{\alpha} \mathcal{U}^{v}\right)\right]
$$

where $\mathcal{G}\left(\frac{1}{z}\right)=\frac{K_{3}\left(\frac{1}{z}\right)}{K_{2}\left(\frac{1}{z}\right)}$.

\section{The Field Contribution to $f^{(1)}$}

The rest of the calculations in this paper focuses on the gravitational field contribution to the heat flux, which arises from the third term on the right hand side of Equation (18). Thus, we isolate such term by defining

$$
f_{\mathcal{U}}^{(1)}=-\tau_{c} \frac{v_{\alpha} f^{(0)}}{z c^{2}}\left(\frac{\partial \mathcal{U}^{\alpha}}{\partial x^{\mu}}+\Gamma_{\mu \nu}^{\alpha} \mathcal{U}^{v}\right) v^{\mu}
$$

In order to establish the corresponding thermodynamic flux, space and time components are separated as follows (Latin indexes run up to three and Greek ones up to four): 


$$
f_{\mathcal{U}}^{(1)}=-\frac{\tau_{c} f^{(0)}}{z c^{2}}\left(v_{\alpha} v^{\ell} \frac{\partial \mathcal{U}^{\alpha}}{\partial x^{\ell}}+v_{\alpha} v^{4} \frac{\partial \mathcal{U}^{\alpha}}{\partial x^{(4)}}+v_{\alpha} v^{\mu} \Gamma_{\mu \nu}^{\alpha} \mathcal{U}^{v}\right)
$$

At this point, the Euler equation is introduced as a requirement in order to guarantee the existence of the Chapman-Enskog solution $[9,10,18]$. This step allows for $f^{(1)}$ to be expressed in terms of the local state variables' gradients. Indeed, since the hydrodynamic velocity satisfies [9]

$$
\tilde{\rho} \dot{\mathcal{U}}^{v}=-h^{v \alpha} p_{, \alpha}
$$

where $\tilde{\rho}$ is $\left(\frac{n \varepsilon}{c^{2}}+\frac{p}{c^{2}}\right)$, with $\varepsilon$ the internal energy per particle, $p$ the local pressure and $h^{v \alpha}$ the spatial projector defined as $\tilde{g}^{v \alpha}+\frac{\mathcal{U}^{v} \mathcal{U}^{\alpha}}{c^{2}}$, we can write:

$$
\mathcal{U}^{(4)} \frac{\partial \mathcal{U}^{v}}{\partial x^{(4)}}=-\frac{h^{v \alpha} p, \alpha}{\tilde{\rho}}-\Gamma_{\mu \beta}^{v} \mathcal{U}^{\beta} \mathcal{U}^{\mu}-\mathcal{U}^{\ell} \frac{\partial \mathcal{U}^{v}}{\partial x^{\ell}}
$$

and thus

$$
f_{\mathcal{U}}^{(1)}=-\frac{\tau_{c} f^{(0)}}{z c^{2}}\left(v_{\alpha} v^{\ell} \frac{\partial \mathcal{U}^{\alpha}}{\partial x^{\ell}}+\frac{v_{\alpha} v^{(4)}}{\mathcal{U}^{(4)}}\left(-\frac{h^{v \alpha} p, \alpha}{\tilde{\rho}}-\Gamma_{\mu \beta}^{v} \mathcal{U}^{\beta} \mathcal{U}^{\mu}-\mathcal{U}^{\ell} \frac{\partial \mathcal{U}^{v}}{\partial x^{\ell}}\right)\right)
$$

In what follows, only the terms depending on Christoffel symbols will be taken into account since they contain the curvature, and thus the gravitational effects:

$$
f_{[g]}^{(1)}=-\frac{\tau_{c} f^{(0)}}{z c^{2}}\left(\frac{-v_{\alpha} v^{(4)}}{\mathcal{U}^{(4)}} \Gamma_{\beta \lambda}^{\alpha} \mathcal{U}^{\lambda} \mathcal{U}^{\beta}+v_{\alpha} v^{\mu} \Gamma_{\mu \nu}^{\alpha} \mathcal{U}^{v}\right)
$$

Also, calculations will be performed in the comoving frame where $\mathcal{U}^{v}=(0,0,0, c)$, $k^{v} \equiv\left(k^{1}, k^{2}, k^{3}, c\right), v_{\eta}=\gamma_{(k)} k_{\eta}$ with $k_{\eta}$ representing the chaotic velocity (the molecule's velocity measured in the comoving frame) and $\gamma_{(k)}=\left(1-\frac{k^{l} k_{l}}{c^{2}}\right)^{-1 / 2}[9,19]$. With these substitutions we obtain the following expression for the gravitational contribution to the first order in the gradients distribution function:

$$
f_{[g]}^{(1)}=-\frac{\tau_{c} \gamma_{(k)}^{2} f^{(0)}}{z c}\left(k_{\alpha} k^{\mu} \Gamma_{\mu 4}^{\alpha}-c k_{\alpha} \Gamma_{44}^{\alpha}\right)
$$

In reference [3], the terms corresponding to the ones in parenthesis in Equation (24) cancel out as the molecular acceleration term is expressed in terms of a force induced by the field, i.e., $\frac{d v^{\mu}}{d \tau}=-\Gamma_{\alpha \beta}^{\mu} v^{\alpha} v^{\beta}$, together with the fact that a partial derivative is considered for the hydrodynamic velocity in Equation (18) of reference [3].

Before proceeding to the heat flux calculation it is useful to notice that the summation $k_{\alpha} k^{\mu} \Gamma_{\mu 4}^{\alpha}$ vanishes up to first order in $\frac{\phi}{c^{2}}$, so that:

$$
f_{[g]}^{(1)}=\frac{\tau_{c} \gamma_{(k)}^{2} f^{(0)}}{z}\left(k_{\alpha} \Gamma_{44}^{\alpha}\right) .
$$

Equation (25) is the basis of the heat flux field contribution that will be calculated in the next section.

\section{Heat Flux Calculation with a Spherically Symmetric Static Metric}

Kinetic theory's definition of heat flux in the comoving frame is expressed as follows [20]:

$$
Q^{\ell}=m c^{2} \int k^{\ell} f^{(1)} \gamma_{(k)}\left(\gamma_{(k)}-1\right) d^{*} K,
$$


where the volume element is $d^{*} K=4 \pi c^{3}\left(\gamma_{(k)}^{2}-1\right)^{\frac{1}{2}} d \gamma_{(k)}$ [19]. Now, using Equation (25) for $f_{[g]}^{(1)}$, the gravitational field contribution to the heat flux can be written as:

$$
Q_{[g]}^{\ell}=\frac{\tau_{c} m c}{z} \int k^{\ell} f^{(0)} \gamma_{(k)}^{3}\left(\gamma_{(k)}-1\right)\left(c k_{\alpha} \Gamma_{44}^{\alpha}\right) d^{*} K
$$

Performing the calculations (see the Appendix A for details) it is obtained that:

$$
Q_{[g]}^{\ell}=\frac{\tau_{c} m n c^{4}}{3 z} \tilde{g}^{\ell \ell} \Gamma_{44}^{\ell}\left[1+5 z \mathcal{G}\left(\frac{1}{z}\right)-\mathcal{G}\left(\frac{1}{z}\right)\right]
$$

In an isotropic Schwarzschild metric, after substitution of the Christoffel symbols, it is found that the gravitational contribution to the heat flux in a simple dilute general relativistic fluid is:

$$
Q_{[g]}^{\ell}=\tau_{c} n k_{B} T\left\{1+5 z \mathcal{G}\left(\frac{1}{z}\right)-\mathcal{G}\left(\frac{1}{z}\right)\right\} \Phi^{, \ell},
$$

A similar expression was obtained for simple charged relativistic fluids in the presence of electrostatic fields [21].

This effect vanishes in the non-relativistic case and is always present in the relativistic regime, both in special and in general relativity, at least in the case of a static and symmetric metric. When the limit $z \rightarrow 0$ (low temperature) is considered one obtains:

$$
Q_{[g]}^{\ell}=\frac{5 z n m \tau_{c} c^{4}}{2} \frac{\Phi^{, \ell}}{c^{2}}=\frac{5 n \tau_{c} k^{2} T^{2}}{2 m} \frac{\Phi^{\ell}}{c^{2}} .
$$

This result is in agreement with the one presented in references [1,2].

\section{Conclusions}

It was shown that a gravitational field contributes to the heat flux of a simple dilute general relativistic fluid. Covariant derivatives were explicitly introduced in Boltzmann's equation for structureless particles that follow geodesic trajectories in a static and isotropic space time. The acceleration term in Boltzmann's equation vanishes if this geodesic approach to the particle dynamics is considered. The expression obtained leads to the low temperature result in which the gravitational contribution to the heat flux corresponds to $\frac{\Phi^{, \ell}}{c^{2}}$ which is negligible in comparison to its thermal (Fourier) counterpart, as expected. The case of a flat space-time in Cartesian coordinates was addressed in reference [2] where the covariant derivative vanishes (Christoffel symbols are zero). In that case, the field coupling with the heat flux would survive if a linearized gravity approach is taken into account.

In contrast with related works, the gravitational field is coupled with the heat flux and will contribute to the entropy production; in other words, the curvature of space-time contributes to the heat flux and produces entropy through the motion of the particles. A complete evaluation of the entropy production $\left(\sigma=-\frac{k_{B}}{c} \int J\left(f f^{\prime}\right) \varphi d^{*} v\right.$, with $\varphi=\frac{\varsigma f^{(1)}}{f^{(0)}}$ and $\varsigma$ the Knudsen parameter) will be addressed in a separate work.

Future work corresponds to the study of tensor effects, which involve viscosity coefficients and the use of Christoffel symbols as thermodynamic forces. In the longer term a similar formalism will be presented to thoroughly analyze the entropy production associated with a microscopic (quantum) approach to the gravitational field.

Acknowledgments: The authors wish to thank Alma Sagaceta-Mejia and Humberto Mondragon-Suarez for their valuable comments to this work and acknowledge support from CONACyT through grant number CB2011/167563.

Author Contributions: The authors contributed equally to this work. 
Conflicts of Interest: The authors declare no conflict of interest.

\section{Appendix A}

In this appendix, details of the gravitational field contribution to the heat flux presented in Section 4 are described. Performing the summation over $\alpha$ in Equation (27) we have:

$$
Q_{[g]}^{\ell}=\frac{\tau_{c} m c^{2}}{z} \int k^{\ell} f^{(0)} \gamma_{(k)}^{3}\left(\gamma_{(k)}-1\right)\left(k_{1} \Gamma_{44}^{1}+k_{2} \Gamma_{44}^{2}+k_{3} \Gamma_{44}^{3}+k_{4} \Gamma_{44}^{4}\right) d^{*} K,
$$

which leads to :

$$
Q_{[g]}^{\ell}=\frac{\tau_{c} m c^{2}}{z} \int f^{(0)} \gamma_{(k)}^{3}\left(\gamma_{(k)}-1\right) \Gamma d^{*} K
$$

where factor $\Gamma$ is:

$$
\Gamma=\left(\begin{array}{l}
\Gamma_{44}^{1} k^{(1)} k_{(1)}+\Gamma_{44}^{2} k^{(1)} k_{(2)}+\Gamma_{44}^{3} k^{(1)} k_{(3)}+\Gamma_{44}^{4} k^{(1)} k_{(4)} \\
\Gamma_{44}^{1} k^{(2)} k_{(1)}+\Gamma_{44}^{2} k^{(2)} k_{(2)}+\Gamma_{44}^{3} k^{(2)} k_{(3)}+\Gamma_{44}^{4} k^{(2)} k_{(4)} \\
\Gamma_{44}^{1} k^{(3)} k_{(1)}+\Gamma_{44}^{2} k^{(3)} k_{(2)}+\Gamma_{44}^{3} k^{(3)} k_{(3)}+\Gamma_{44}^{4} k^{(3)} k_{(4)}
\end{array}\right)
$$

In order to write Equation (A2) in covariant form, it will be used that $\tilde{g}^{\ell \ell} k_{(\ell)}=k^{(\ell)}$ for $\ell=1,2,3$, and thus $k^{(1)} k_{(1)}=\left(k^{(1)}\right)^{2}$. Taking into account that all the terms with the factor $k^{(\mu)} k_{(v)}$ for $\mu \neq v$ vanish for parity reasons, we shall have:

$$
Q_{[g]}^{\ell}=\frac{\tau_{c} m c^{2}}{z} \int f^{(0)} \gamma_{(k)}^{3}\left(\gamma_{(k)}-1\right)\left(\begin{array}{c}
\Gamma_{44}^{1} \tilde{g}^{11}\left[k^{(1)}\right]^{2} \\
\Gamma_{44}^{2} \tilde{g}^{22}\left[k^{(2)}\right]^{2} \\
\Gamma_{44}^{3} \tilde{g}^{33}\left[k^{(3)}\right]^{2}
\end{array}\right) d^{*} K
$$
written as:

Since the three integrals are equal and $\left[k^{(1)}\right]^{2}+\left[k^{(2)}\right]^{2}+\left[k^{(3)}\right]^{2}=k^{2}$, Equation (A4) can be

$$
Q_{[g]}^{\ell}=\frac{\tau_{c} m c^{2}}{3 z} \int f^{(0)} \gamma_{(k)}^{3}\left(\gamma_{(k)}-1\right) k^{2} \tilde{g}^{\ell \ell} \Gamma_{44}^{\ell} d^{*} K
$$

The next step is to express the integral in terms of $\gamma_{(k)}$ :

$$
Q_{[g]}^{\ell}=\frac{\tau_{c} m c^{2}}{3 z} \int_{1}^{\infty} f^{(0)} \gamma_{(k)}^{3}\left(\gamma_{(k)}-1\right) \frac{c^{2}\left(\gamma_{(k)}^{2}-1\right)}{\gamma_{(k)}^{2}} \tilde{g}^{\ell \ell} \Gamma_{44}^{\ell} 4 \pi c^{3}\left(\gamma_{(k)}^{2}-1\right)^{\frac{1}{2}} d \gamma_{(k)},
$$

and after Juttner's distribution function is substituted we obtain that:

$$
Q_{[g]}^{\ell}=\frac{\tau_{c} m n c^{4}}{3 z K_{2}\left(\frac{1}{z}\right)} \tilde{g}^{\ell \ell} \Gamma_{44}^{\ell} \int_{1}^{\infty} e^{-\frac{\gamma_{(k)}}{z}} \gamma_{(k)}\left(\gamma_{(k)}-1\right)\left(\gamma_{(k)}^{2}-1\right)^{\frac{3}{2}} d \gamma_{(k)},
$$

from where the field contribution to the heat flux is given by Equation (28):

$$
Q_{[g]}^{\ell}=\tau_{c} n k_{B} T\left\{1+5 z \mathcal{G}\left(\frac{1}{z}\right)-\mathcal{G}\left(\frac{1}{z}\right)\right\} \Phi^{, \ell},
$$

where the substitution of $\tilde{g}^{\ell \ell} \Gamma_{44}^{\ell}$ values has been performed. 


\section{References}

1. Tolman, R.C. On the weight of heat and thermal equilibrium in general relativity. Phys. Rev. 1930, 35, 904-924.

2. Sandoval-Villalbazo, A.; Garcia-Perciante, A.L.; Brun-Battistini, D. Tolman's law in linear irreversible thermodynamics: A kinetic theory approach. Phys. Rev. D 2012, 86, doi:10.1103/PhysRevD.86.084015.

3. Kremer, G.M. Relativistic gas in a Schwarzschild metric. J. Stat. Mech. 2013, 4, P04016-P04026.

4. Weinberg, S. Gravitation and Cosmology: Principles and Applications of the General Theory of Relativity; John Wiley \& Son: New York, NY, USA, 1972.

5. Wodarzik, U.F. Spherically symmetric heat conduction in general relativity. Phys. Rev. A 1984, 30, 3-7.

6. Eckart, C. The Thermodynamics of Irreversible Processes III: Relativistic Theory of the Simple Fluid. Phys. Rev. 1940, 58, 919-930.

7. Hiscock, A.; Lindblom, L. Generic instabilities in first order dissipative relativistic fluid theories. Phys. Rev. D 1985, 31, 725-740.

8. De Groot, S.R.; van Leeuwen, W.A.; van der Wert, C. Relativistic Kinetic Theory; North Holland Publishing Co.: Amsterdam, The Netherlands, 1980.

9. Cercignani, C.; Kremer, G.M. The Relativistic Boltzmann Equation: Theory and Applications; Birkhauser Verlag: Basel, Switzerland, 2002.

10. Chapman, S.; Cowling, T.G. The Mathematical Theory of Non-Uniform Gases, 2nd ed.; Cambridge Mathematical Library: Cambridge, UK, 1953.

11. Kremer, G.M. The Boltzmann equation in special and general relativity. In Proceedings of the 28th International Symposium on Rarefied Gas Dynamics, Zaragoza, Spain, 9-13 July 2012.

12. Crothers, S.J. On isotropic coordinates and Einstein's gravitational field. Prog. Phys. 2006, 3, 7-12.

13. Rybicki, G.; Lightman, A. Radiative Processes in Astrophysics, 1st ed.; John Wiley and Sons: New York, NY, USA, 1979.

14. Kolb, E.; Turner, M. The Early Universe, 1st ed.; Addison-Wesley Publishing Company: Reading, MA, USA, 1993.

15. Jüttner, F. Das Maxwellsche Gesetz der Geschwindigkeitsverteilung der Relativtheorie. Ann. Phys. Chem. 1911, 34, 856-860.

16. Cubero, D.; Casado-Pascual, J.; Dunkel, J.; Talkner, P.; Hänggi, P. Thermal Equilibrium and Statistical Thermometers in Special Relativity. Phys. Rev. Lett. 2007, 99, doi:10.1103/PhysRevLett.99.170601.

17. Bhatnagar, P.L.; Gross, E.P.; Krook, M. A Model for Collision Processes in Gases I. Small Amplitude Processes in Charged and Neutral One-Component Systems. Phys. Rev. 1954, 94, 511, doi:10.1103/PhysRev.94.511.

18. Courant, R.; Hilbert, D. Methods of Mathematical Physics, 1st ed.; John Wiley and Sons: New York, NY, USA, 1989.

19. Garcia-Perciante, A.L.; Mendez, A.R. Heat conduction in relativistic neutral gases revisited. Gen. Relativ. Gravit. 2011, 43, 2257-2267.

20. Garcia-Perciante, A.L.; Sandoval-Villalbazo, A.; Garcia-Colin, L.S. On the microscopic nature of dissipative effects in special relativistic kinetic theory. J. Non-Equilib. Thermodyn. 2012, 37, 43-61.

21. Garcia-Perciante, A.L.; Sandoval-Villalbazo, A.; Garcia-Colin, L.S. Benedicks effect in a relativistic simple fluid. J. Non-Equilib. Thermodyn. 2013, 38, 141-150.

(c) 2017 by the authors. Licensee MDPI, Basel, Switzerland. This article is an open access article distributed under the terms and conditions of the Creative Commons Attribution (CC BY) license (http://creativecommons.org/licenses/by/4.0/). 